\title{
Active Contour Based on Local Statistic Information and an Attractive Force for Ultrasound Image Segmentation
}

\author{
Jianjun Yuan* and Jianjun Wang \\ School of Mathematics and Statistics, Southwest University, Chongqing, China \\ ${ }^{*}$ Corresponding author
}

\begin{abstract}
This paper presents a new active contour model with local intensities through level set method for ultrasound images segmentation. The method is not affected by the limitation of Gaussian distribution. The model is designed by local intensities, alignment term with a sharpening edge coefficient and regularization. Local intensities have the capability of denoising, and local means and variances are considered. The alignment term with a sharpening edge coefficient can sharpen edge and increase the convergence speed. The numerical schedule is implemented by level set method. Experimental results show that proposed method succeed to segment edges for ultrasound images.
\end{abstract}

Keywords-image segmentation; local statistic; level set; regularization

\section{INTRODUCTION}

Image segmentation plays a key role in image processing. Accurate segmentation has many applications such as medical images ${ }^{[1-10]}$. However, owing to noise, low contrast and intensity inhomogeneity still deserve research ${ }^{[11,12]}$, for example, edge detection and thresholding ${ }^{[4]}$.

Active contours ${ }^{[13]}$ enables closed and smooth edges to be obtained. The existing active contour model methods have the methods of edge-based ${ }^{[13-15]}$ and region-based ${ }^{[10,12,16-22]}$. They have own advantages and disadvantages.

In these models, Gaussian distribution is assumed. However, intensity distribution in an image does not always meet the Gaussian distribution. The assumption limits its application.

In this paper, a new segmentation model using variational level set for ultrasound image segmentation is proposed. A kernel function with localization, local intensity means and variances are utilized. An alignment term with a sharpening coefficient is introduced. It can extract object boundaries more accurately. Simulation experiments show that segmentation accuracy is improved.

The context is organized: Introduction to proposed method in section 2, Section 3 shows experimental results, Conclusion is drawn in Section 5.

\section{THE PROPOSED MODEL}

The whole energy function has the following parts: local intensity $E^{N L I}$, alignment term $E^{A T}$, and the regularization component $E^{S}$, they are defined as follows.

\section{A. New Local Intensity Information}

Let $\Omega \in R^{2}$ be an image domain, and $I: \Omega \rightarrow R$ a given gray level image, $\phi$ is a level set function. For $x \in \Omega$, a new local intensity (NLI) model can be written :

$$
E_{x}^{N L I}\left(\phi, d_{i}\right)=\sum_{i=1}^{n} \lambda_{i} \int_{\Omega_{i}} K_{\sigma}(x-y)\left|I(y)-d_{i}(x)\right|^{2} d y
$$

with

$$
d_{i}(x)=c_{i}(x)+5 \operatorname{sign}\left(I(y)-c_{i}(x)\right) \sigma_{i}(x)
$$

where $c_{i}$ denotes local mean, $\sigma_{i}$ is local standard variance of the $i$ th region. $\lambda_{i}$ is weight parameter. sign represents the sign function. $C_{1}, C_{2}$ can be worked out:

$$
\left\{\begin{array}{c}
c_{1}=\frac{\int K_{\sigma} I(x) H(\phi) d x}{\int K_{\sigma} H(\phi) d x} \\
c_{2}=\frac{\int K_{\sigma} I(x)(1-H(\phi)) d x}{\int K_{\sigma}(1-H(\phi)) d x}
\end{array}\right.
$$

$\sigma_{1}^{2}, \sigma_{2}^{2}$ can be gotten:

$$
\left\{\begin{array}{c}
\sigma_{1}^{2}=\frac{\int K_{\sigma}\left(I(x)-c_{1}\right)^{2} H(\phi) d x}{\int K_{\sigma} H(\phi) d x} \\
\sigma_{2}^{2}=\frac{\int K_{\sigma}\left(I(x)-c_{2}\right)^{2}(1-H(\phi)) d x}{\int K_{\sigma}(1-H(\phi)) d x}
\end{array}\right.
$$


$H(\phi)$ is Heaviside function. The Gaussian kernel $K_{\sigma}(x-y)$ decreases drastically to zero as $y$ goes away from $x$. It can be controlled by the scale parameter $\sigma>0$. To compute the convolutions more efficiently, the kernel $K_{\sigma}$ is truncated as a $(2 r+1) \times(2 r+1)$ mask, where $r$ is not less than $2 \sigma$. If $I(y)>c_{i}(x), d_{i}(x)=c_{i}(x)+5 \sigma_{i}(x)$. The energy will drive the evolving curve to move towards the position $c_{i}(x)+5 \sigma_{i}(x)$. It has the effect of dilating. If $I(y)=c_{i}(x), d_{i}(x)=c_{i}(x)$, the evolving curve will stop at the position $c_{i}(x)$. Otherwise, $I(y)<c_{i}(x), d_{i}(x)=c_{i}(x)-5 \sigma_{i}(x)$, the energy will drive the evolving curve to move towards the position $c_{i}(x)-5 \sigma_{i}(x)$. It has the effect of shrinking. The intuitive illustrations of the method are shown in Fig.1 (a) - (b).

To sum up, the NLI energy function can be represented as

$$
E^{N L I}=\int_{\Omega} E_{x}^{N L I}\left(d_{1}, d_{2}\right) d x
$$

\section{B. Alignment Term with a Sharpening Edge Coefficient}

The alignment term can increase the attraction force. The sharpening edge coefficient can enhance weak boundaries. The robust alignment term with a sharpening edge coefficient can be defined as:

$$
E^{A T}(\phi)=b\left(|\nabla I|^{2}\right) \oint_{C}|\langle\nabla g, N\rangle| d s
$$

Where $\nabla$ denotes the gradient operator, $\langle\cdot, \cdot\rangle$ is inner product in $R^{2} . N$ represents outwards normal vector of $C$. $d s$ is arc length differential. The function $g$ denotes an edge detector function, and $g:[0,+\infty) \rightarrow[0,+\infty)$ satisfies: (1) $g$ is a regular monotonic decreasing function; (2) $g(0)=1$, $\lim _{s \rightarrow+\infty} g(s)=0$. In this paper, a typical choice of $g$ is $g(s)=1 /(1+s / T), T$ is a given threshold. For sharpening weak edges, we introduce a sharpening edge coefficient. It can enhance the segmentation of some weak edges. The sharpening edge coefficient is defined as:

$$
b(u)=2 u g^{\prime}(u)+g(u)
$$

In this paper, let $u=|\nabla I|^{2}$, so gradient descend flow equation

$$
\frac{\partial \phi}{\partial t}=b\left(|\nabla I|^{2}\right) \operatorname{sign}(\langle\nabla g, \nabla \phi\rangle) \Delta g
$$

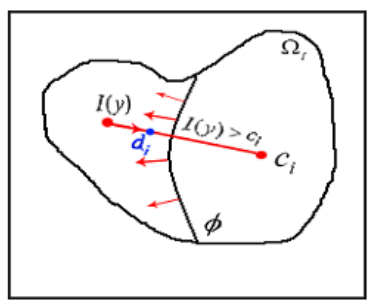

(a)

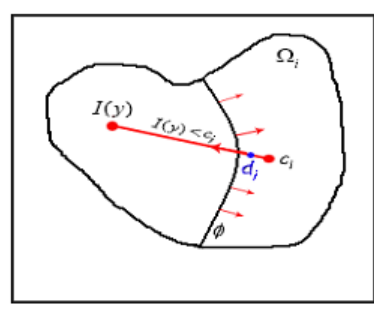

(b)
FIGURE I. THE INTUITIVE ILLUSTRATIONS. (A) THE ILLUSTRATION OF EXPANDING FROM MEAN. (B) THE ILLUSTRATION OF SHRINKING TOWARDS MEAN

\section{Regularization Term}

In this part, a regularization ${ }^{[14]}$ is proposed, and characterized by the following energy function:

$$
P(x)=\frac{1}{2} \int_{\Omega}(|\nabla \phi|-1)^{2} d x
$$

In addition, a level set of penalizing contour length is introduced:

$$
L(\phi)=\int_{\Omega}|\nabla H(\phi)| d x
$$

Therefore, the entire regularization component $E^{S}$ energy function is

$$
E^{S}(\phi)=\mu P(\phi)+v L(\phi)
$$

where $\mu, v>0$ are weighting constants.

\section{Level Set Formulation}

The integrated level set formulation is

$$
\begin{aligned}
\frac{\partial \phi}{\partial t}= & -\alpha \delta_{\varepsilon}(\phi)\left(\lambda_{1} e_{1}-\lambda_{2} e_{2}\right) \\
& +\beta b\left(|\nabla I|^{2}\right) \operatorname{sign}(\langle\nabla g, \nabla \phi\rangle) \Delta g \\
& +\mu\left(\Delta \phi-\operatorname{div}\left(\frac{\nabla \phi}{|\nabla \phi|}\right)\right)+v \delta_{\varepsilon}(\phi) \operatorname{div}\left(\frac{\nabla \phi}{|\nabla \phi|}\right)
\end{aligned}
$$

with

$$
\left\{\begin{array}{l}
e_{1}(x)=\int_{\Omega_{i}} K_{\sigma}(x-y)\left[I(y)-d_{1}(x)\right]^{2} d y \\
e_{2}(x)=\int_{\Omega_{i}} K_{\sigma}(x-y)\left[I(y)-d_{2}(x)\right]^{2} d y
\end{array}\right.
$$

\section{EXPERIMENTAL RESULTS}

The performances of proposed method are tested. All experiments are performed on a $2.4 \mathrm{GHz}$ Intel(R) Core(TM)2 Duo CPU PC with 4G memory. In this section, some parameters are set as follows: $c_{0}=2$, time step $\Delta t=0.1$, 
$\alpha=0.1, \beta=0.01, \mu=1.0, \lambda_{1}=1.0, \lambda_{2}=1.0$ or 2.0 , $v=0.01 \times 255^{2}$ or $0.08 \times 255^{2}$. The values of $\sigma$ should be chosen carefully. Too small $\sigma$ or too large $\sigma$ may cause an undesirable result. It can be chosen according to the effect of noise in an image. In all the following experiments, we take $\sigma=2.0,3.0,5.0$.

\section{A. Segmentation of Synthetic Images}

To test the performance of proposed method, we apply it to synthetic images with weak boundary, intensity inhomogeneity and different levels of speckle noise.

From Fig.2, it can be seen that the proposed method successfully extract the object boundary. The LBF model fails. In Fig.2(c), the cross-sections of the middle rows of the object boundary are showed. The result of our method is approximated to the true boundary. The derivation is larger between the result of the LBF model and the true boundary.

Secondly, due to the intensities distribution of a ultrasound image following the Rayleigh distribution ${ }^{[23]}$, in this paper, the different level speckle noise following the Rayleigh distribution are added to original images, respectively. In Fig.3, the synthetic images are corrupted by speckle noise with different levels ( $\sigma=5,10,15,20)$. We compare the three existing methods with our method. LBF ${ }^{[11]}$ and $\mathrm{LIF}^{[12]}$ are sensitive to noise. Under the hypothesis condition of following Gaussian distribution, the LGD model ${ }^{[21]}$ is insensitive to speckle noise, but it extracts the shadow, and the result is inaccurate. The proposed method has the capability of antinoise and also can segment the boundary successfully.

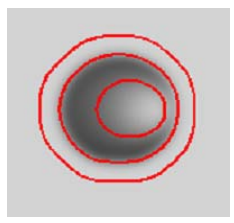

(a)

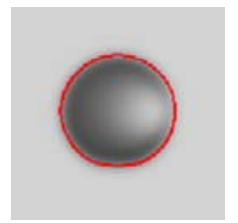

(b)

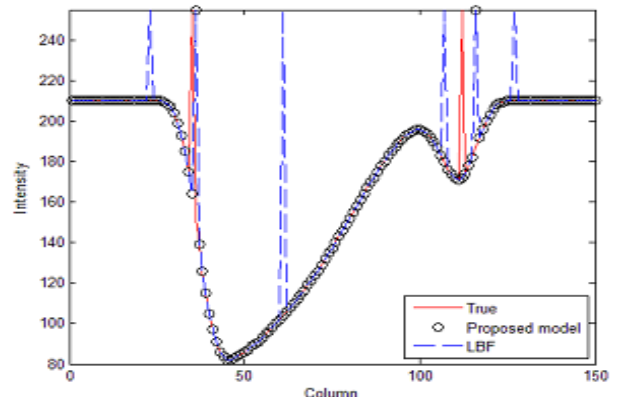

(c) show by quantitative comparison that proposed method segments more contours than the other methods. Since the accurate position of object and background regions of the synthetic images are precisely known in advance as shown in the first row image in Fig.3, it is rational to use the root mean squared error (RMSE) for evaluating the performance. The RMSE measures a distance between the segmented contours and the exact object boundary. Then the RMSE is computed as follows:

$$
R M S E=\sqrt{\frac{\sum_{k=0}^{n-1}\left[\left(x_{k}-\bar{x}_{k}\right)^{2}+\left(y_{k}-\bar{y}_{k}\right)^{2}\right]}{n}}
$$

The lower RMSE means that there are fewer pixels misclassified, i.e., the image can be segmented more accurately. The proposed method is applied to synthetic images with different levels of noise in Fig.3. Additionally, for comparison, the over three methods are also applied to the same images. From Table.1, we can see that the RMSE values of our method are the least than that of the others.
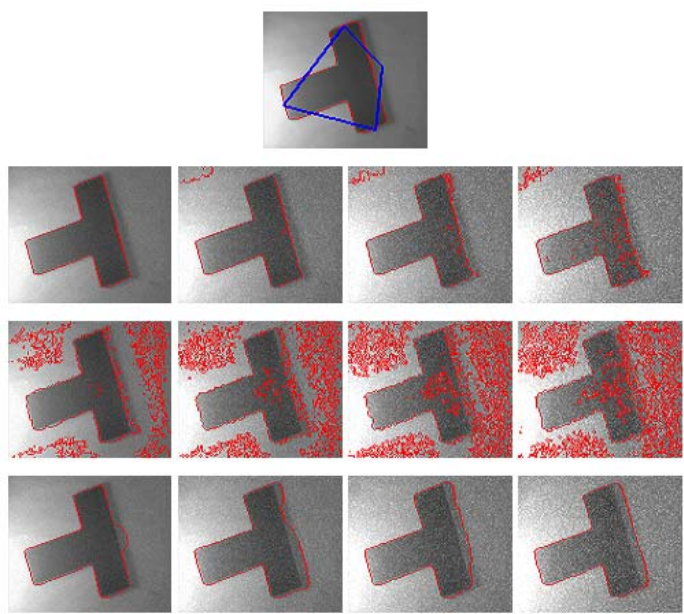

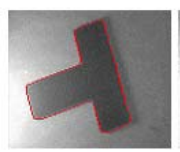

(a)

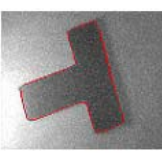

(b)

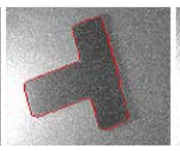

(c)

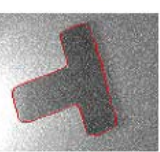

(d)
FIGURE III. COMPARISON WITH FOUR DIFFERENT METHODS FOR SYNTHETIC IMAGES WITH DIFFERENT LEVEL NOISE (FROM (A) TO (D) COLUMN: $\sigma=5,10,15,20$ ) AND INTENSITY INHOMOGENEITY. 1ST ROW: ORIGINAL IMAGE WITH AN INITIAL CONTOUR AND TRUE BOUNDARY; 2ND ROW: RESULTS OF THE LBF MODEL; 3RD ROW: RESULTS OF THE LIF MODEL; 4TH ROW: RESULTS OF THE LGD MODEL; 5TH ROW: RESULTS OF OUR METHOD

FIGURE II. COMPARISON WITH TWO DIFFERENT METHODS. (A) RESULT OF THE LBF MODEL; (B) RESULT OF THE PROPOSED MODEL; (C) CROSS-SECTIONS OF THE MIDDLE ROWS OF THE TRUE BOUNDARY (RED), THE SEGMENTATIONS OF THE PROPOSED METHOD (BLACK) AND THE LBF MODE L (BLUE)

For comparison, our method has better capability of dealing with noisy image than the over three models in Fig.3. We can 
TABLE I. THE RMSES OF THE SEGMENTATION RESULTS OF SYNTHETIC IMAGES WITH DIFFERENT LEVEL NOISE

\begin{tabular}{|c|c|c|c|c|}
\hline \multirow{2}{*}{$\begin{array}{c}\text { Noise } \\
\text { level }\end{array}$} & \multicolumn{4}{|c|}{ RMSE } \\
\cline { 2 - 5 } & LBF & LIF & LGD & $\begin{array}{c}\text { Proposed } \\
\text { method }\end{array}$ \\
\hline 5 & 1.05 & 27.64 & 5.81 & 0.83 \\
\hline 10 & 6.77 & 37.02 & 11.38 & 1.41 \\
\hline 15 & 9.63 & 45.93 & 13.62 & 1.94 \\
\hline 20 & 17.87 & 53.05 & 16.84 & 2.23 \\
\hline
\end{tabular}

\section{B. Segmentation of Several Real Images}

Fig.4 presents the segmented results for real ultrasound images. From Fig.4, LBF and LIF model fail to segment. The local means only are considered in the two models, the local means cannot reflect accurately the change of intensities, and it also is sensitive to noise. The local means and variances are considered in the LGD model, it has the capability of segmenting some ultrasound images. However, the assumption of the LGD model is that the intensities follow the Gaussian distribution. In addition, the computational cost is large by the LGD model, and it can appear over-segmentation for weak boundaries. For example, the results of the third and fourth rows in Fig.4(c) appear over-segmentation. The LGD method also fails to extract multiple object boundaries for the image of the final row in Fig.4(c). The proposed model succeeds to segment the boundaries, and the over-segmentation does not appear.

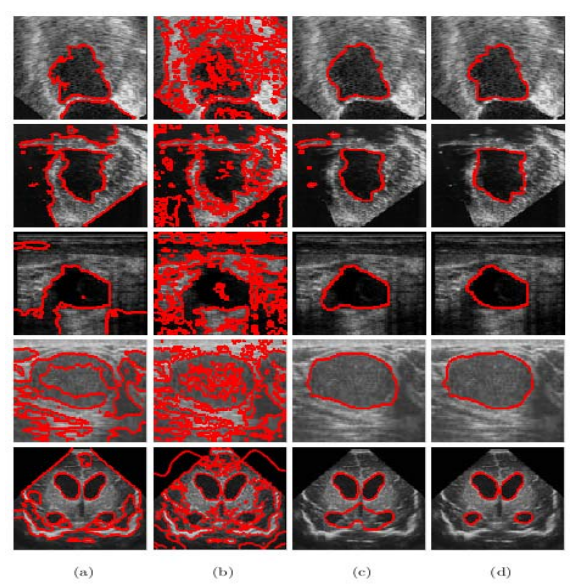

FIGURE IV. RESULTS COMPARISON FOR REAL ULTRASOUND IMAGES WITH THE FOUR DIFFERENT METHODS. (A) RESULTS OF THE LBF MODEL; (B) RESULTS OF THE LIF METHOD; (C) RESULTS OF THE LGD MODEL; (D) RESULTS OF OUR MODEL

\section{Computational Time and Iterations}

The proposed method requires relatively high computational complexity. Table. 2 presents the processing speeds of the four methods. All the four methods were performed on a $2.4 \mathrm{GHz}$ Intel(R) Core(TM) 2 Duo CPU PC with 4G memory using MATLAB. From Table.2, the proposed method requires more time to reach the satisfied results. The main reason is that the proposed method cost more time using the convolution between an image and the kernel function $K_{\sigma}$. Additionally, local means and variances are computed. Computing the second term still need time. However, the iterations are less than the other three methods. The alignment term with a sharpening edge coefficient has the capabilities of sharpening edge and fast convergence.

TABLE II. COMPARISON WITH ITERATIONS AND COMPUTATIONAL TIME FOR THE IMAGES IN FIG.4 FROM TOP TO BOTTOM

\begin{tabular}{|c|l|c|c|c|c|c|}
\hline \multirow{3}{*}{ Method } & & Image1 & Image2 & Image3 & Image4 & Image5 \\
\cline { 2 - 7 } & $95 \times 93$ & $90 \times 100$ & $78 \times 80$ & $127 \times 80$ & $127 \times 97$ \\
\hline \multirow{4}{*}{ LBF } & $\sigma$ & 5.0 & 3.0 & 3.0 & 3.0 & 3.0 \\
\cline { 2 - 7 } & Iteration & 250 & 250 & 250 & 300 & 300 \\
\cline { 2 - 7 } & Time & 3.54 & 3.70 & 3.16 & 4.13 & 4.27 \\
\hline \multirow{4}{*}{ LIF } & $\sigma$ & 5.0 & 5.0 & 5.0 & 5.0 & 5.0 \\
\cline { 2 - 7 } & Iteration & 300 & 300 & 300 & 300 & 300 \\
\cline { 2 - 7 } & Time & 8.16 & 1.09 & 5.69 & 8.84 & 10.45 \\
\hline \multirow{4}{*}{$\begin{array}{c}\text { OGD } \\
\text { method }\end{array}$} & $\sigma$ & 5.0 & 5.0 & 5.0 & 5.0 & 5.0 \\
\cline { 2 - 7 } & Iteration & 250 & 500 & 250 & 300 & 550 \\
\cline { 2 - 7 } & Time & 3.61 & 6.99 & 2.95 & 4.64 & 18.23 \\
\cline { 2 - 7 } & Iteration & 150 & 100 & 100 & 150 & 120 \\
\cline { 2 - 7 } & Time & 6.83 & 3.41 & 1.83 & 7.94 & 3.92 \\
\hline
\end{tabular}

\section{CONCLUSION}

In this paper, a new level set-based active contour model is proposed for ultrasound image segmentation. In this model, an energy function is formulated based on the new local intensity information and an alignment term with a sharpening edge coefficient. The energy function is computed through gradient descent flow to accomplish the segmentation task. Experimental results show that the proposed method can extract accurately the boundaries. Segmentation is compared with the results of the other three models. The RMSE values show the proposed method produces much better segmentation. The processing speeds of the proposed method and the other three methods are also studied. The speed of the proposed method is slightly slower than that of the other three methods. However, the accuracy of the proposed method is the highest and iterations are the least.

\section{ACKNOWLEDGMENT}

The authors are grateful to the anonymous reviewers and the associate editor for their valuable comments, which have greatly helped us to improve this work. This work is supported by Project funded by China Postdoctoral Science Foundation (No. 2016M602625) and Doctoral Funds of Southwest University under contract SWU112113.

\section{REFERENCES}

[1] A. Noble, D. Boukerroui, Ultrasound image segmentation: a survey, IEEE Trans. Med. Imag., 2006, 25(8), 987-1010.

[2] D. Boukerroui, A. Baskurt, J. A. Noble, O. Basset, Segmentation of ultrasound images multi resolution 2D and 3D algorithm based on global and local statistics, Pattern Recognition Lett., 2003, 24, 779-790. 
[3] Yan Chen, Ruming Yin, Patrick Flynn, Shira Broschat, Aggressive region growing for speckle reduction in ultrasound images, Pattern Recognition Lett., 2003, 24, 677-691.

[4] Y. L. Huang, D. R. Chen, Watershed segmentation for breast tumor in 2D sonography, Ultrasound Med. Biol., 2004, 30(5), 625-632.

[5] Zafer Iscan, Mehmet Nadir Kurnaz, Zumray Dokur, Tamer Olmez, Ultrasound Image Segmentation by Using Wavelet Transform and SelfOrganizing Neural Network, Neural Information Processing-Letters and Reviews, Aug.-Sept., 2006, 10(8-9), 183-191.

[6] C. M. Chen, Y. H. Chou, C. S. K. Chen, J. Z. Cheng, Y. F. Ou, F. C. Yeh, K. W. Chen, Cell-competition : a new segmentation algorithm for multiple objects with irregular boundaries in ultrasound images, Ultrasound Med. Biol., 2005, 31(12), 1647-1664.

[7] Kolar, Radim and Kozumplik, Jiri, Fuzzy approach in ultrasound image segmentation, Proceedings of the International Conference, 7th Fuzzy Days on Computational Intelligence, Theory and Applications, SpringerVerlag, London, UK, 2001, 924-929.

[8] C. M. Chen, H. H. S. Lu, Y. C. Lin, A nearly vision-based snake model for ultrasound image segmentation, Ultrasound Med. Biol., 2000, 26(2), 273-285.

[9] D. R. Chen, R. F. Chang, W. J. Wu, W. K. Moon, W. L. Wu, 3D Breast ultrasound segmentation using active contour model, Ultrasound Med. Biol., 2003, 29(7), 1017-1026.

[10] G. Slabaugh, G. Unal, T. Fang, M. Wels, Ultrasound-specific segmentation via decorrelation and statistical region-based active contours, CVPR, June, 2006, 45-53.

[11] C. M. Li, C. Y. Kao, J. C. Gore, Z. H. Ding, Implicit active contours driven by local binary fitting energy, in: Proceedings of the CVPR'07, 2007, 1-7.

[12] K. Zhang, et al., Active contours driven by local image fitting energy, Pattern Recognition, 2010, 4(43), 1199-1206.

[13] Kass, M., Witkin, A., Terzopoulos, D., Snakes: active contour models. Int. J. Comp. Vis., 1987, 1(4), 321-331.

[14] V. Caselles, R. Kimmel, G. Sapiro, On geodesic active contours, Int. J. Comput. Vision, 1997, 22(1), 61-79.

[15] Xu, C., Prince, J., Snakes, shapes, and gradient vector flow. IEEE Trans. Imag. Proc., 1998,7(3), 359-369.

[16] T. Chan, L. Vese, Active contours without edges, IEEE Trans. Image Process., 2001, 10(2), 266-277.

[17] H. Mao, H. Liu, P. Shi, Neighbor-constrained active contours without edges, CVPR, 2008, 23-28 June, 1-7.

[18] R. Ronfard, Region-based strategies for active contour models, Int. J. Comput. Vis., 1994, 13(2),229-251.

[19] Li Wang, Chunming Li, Quansen Sun, Deshen Xia, Chiu-Yen Kao, Active contours driven by local and global intensity fitting energy with application to brain MR image segmentation, Computerized Medical Imaging and Graphics 2009, 33, 520-531.

[20] Li C., Kao C., Gore J. C., Ding Z., Minimization of region-scalable fitting energy for image segmentation. IEEE Trans Image Process, 2008, 17(10), 1940-1949.

[21] Li Wang, Li He, Arabinda Mishra, Chunming Li, Active contours driven by local Gaussian distribution fitting energy, Signal Processing, 2009, 89, 2435-2447.

[22] Bo Liu, H. D. Cheng, Jianhua Huang, Jiawei Tian, Xianglong Tang, Jiafeng Liu, Probability density difference-based active contour for ultrasound image segmentation, Pattern Recognition, 2010, 43, 20282042.

[23] R.F. Wagner, S.W. Smith, J.M. Sandrik, H. Lopez, Statistics of speckle in ultrasound B-scans, IEEE Trans. Sonics Ultrason., 1983, 30(3), 156163. 